\title{
Viper bite in a lebanese child
}

\author{
Abstract \\ Snakebites account for more than 5 million cases around the world, with fatal and deadly \\ consequences.
}

Case: We report a case of a pediatric patient affected by snakebite in North Lebanon and almost recovered.

Discussion: Pediatric snakebites are a serious case of morbidity and mortality. The old steps used in the management have changed and the time of antivenom introduction is very important.

Conclusion: Although snakebites and inadequate management can cause fatal consequences, giving the antivenin at the right time and doing fasciotomy can be lifesaving.

Keywords: akkar, snakebite, lebanese child, venomous, antivenom
Volume 5 Issue 8 - 2016

\author{
El Helou Sally,' Feghali Michel, ${ }^{2}$ Sacy Robert \\ 'Department of Pediatrics, Saint Georges Hospital University \\ Medical Center, University of Balamand, Quarantina \\ governmental hospital, Lebanon \\ ${ }^{2}$ Vascular Surgeon, Saint-George Hospital, University Medical \\ Center, Balamand University and Quarantina Governmental \\ hospital, Lebanon \\ ${ }^{3}$ Department of Pediatrics, Saint Georges Hospital University \\ Medical Center, University of Balamand, Lebanon
}

Correspondence: Robert Sacy, Professor of Pediatrics, Department of Pediatrics, Saint Georges Hospital University Medical Center, University of Balamand, Head of department, Carlos Slim pediatric Center, Quarantina Governmental hospital, Beirut, Lebanon, Email robertsacy@gmail.com 2016

\section{Introduction}

Snakebites account for more than 5 million cases around the world; where near 100000 develop severe sequelae. ${ }^{1}$ A nation's demographics are highly influenced by the pediatric snakebite mortality and morbidity. ${ }^{2}$ Depending on the snake specie, the complications may be life threatening. It is rarely seen in children since usually it is related to agricultural occupations.

The treatment was formulated in guidelines published by the WHO/SEARO. ${ }^{3}$ Here we report a case of a pediatric child affected by a Vipera palaestinabite in North Lebanon and almost recovered.

\section{Case report}

A three year old female patient, previously healthy, who presented to the Quarantine hospital, Beirut, Lebanon after being bitten with a snake two days previous. The girl was in the family garden in Akkar when a black snake bit her on the upper third of the lateral side of her left leg. The parents applied a tourniquet around the girl's leg, excised the wound and suctioned the venom. However, when the girl's leg became swollen and edematous, she was admitted to a hospital in Akkar, where she received two doses of anti-venom and tetanus vaccine. The patient started to vomit and her leg worsens so she was transferred to the Quarantine hospital after three days of antibiotics treatment with no improvement but deterioration.

Upon admission, her left leg was cyanotic, pulseless, cold and edematous, with decreased motility. The cyanosis reached up to the upper chest. The girl became hypotensive and pale so an urgent fasciotomy was done (Figure 1). Broad-spectrum antibiotics and anticoagulant were started. The patient received post op blood transfusion for a hemoglobin of 6.1 and platelet transfusion for a count of 17 000. DIC panel was normal. She also received human albumin since she was kept NPO for several days since she was in pain from her abdominal bruises and due to her wound. Daily dressing changes were done. Debridement was performed twice in the OR. The patient was discharged home when her wound improved after one month of daily dressings.
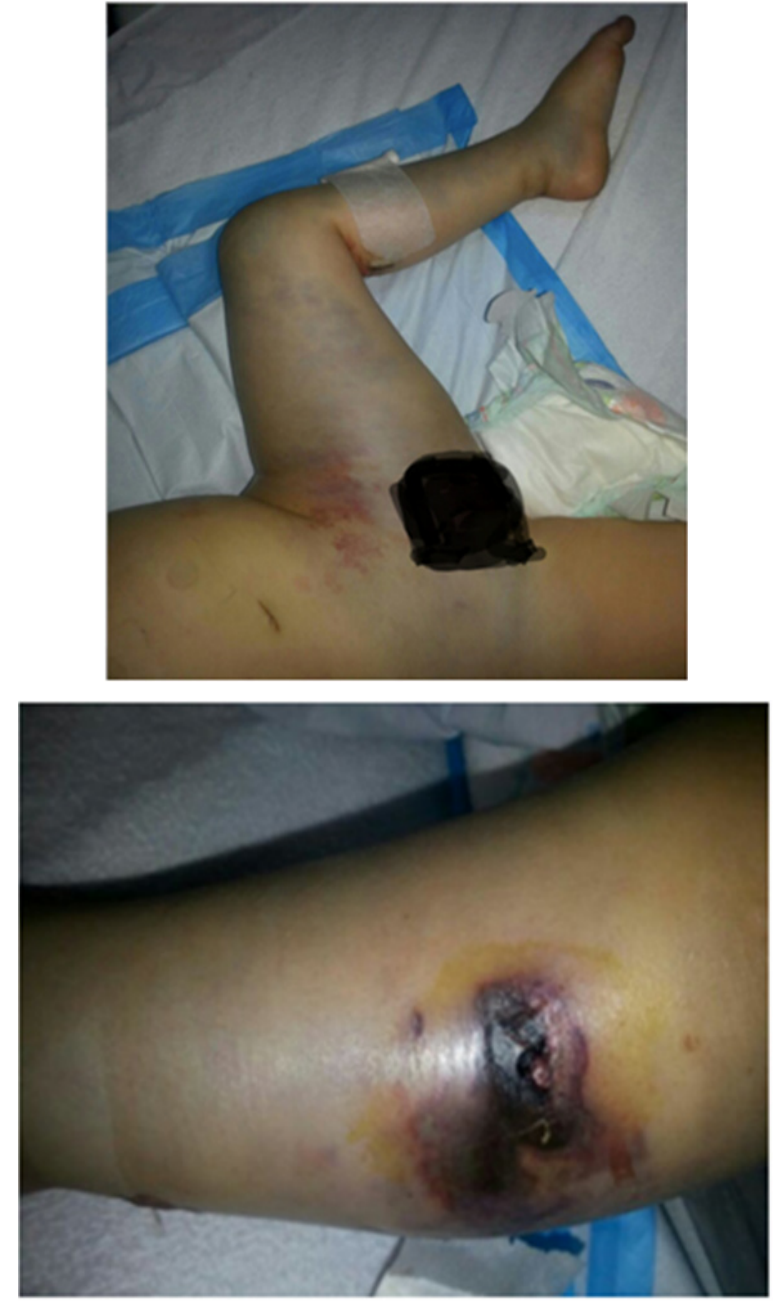

Figure I On admission. 


\section{Discussion}

Pediatric snakebites are not well studied and statistics are not available, but it is a serious cause of morbidity because of the bigger exposure of the venom based on surface area. ${ }^{4}$ The clinical outcome of snakebites differs according to several criteria including the snake specie, size, and venom volume, as well as the age, size and health of the victim. ${ }^{5}$

Snakes are classified into three main families; Elapidae, the Viperidae, and the Hydrophidae. They can be either venomous and cause life threatening events (nephrotoxicity, coagulopathy, neurotoxicity) or nonvenomous. ${ }^{6}$

Our patient was bitten by Vipera palaestina (Figure 2), from the viper family, which is a poisonous specie and can be fatal.

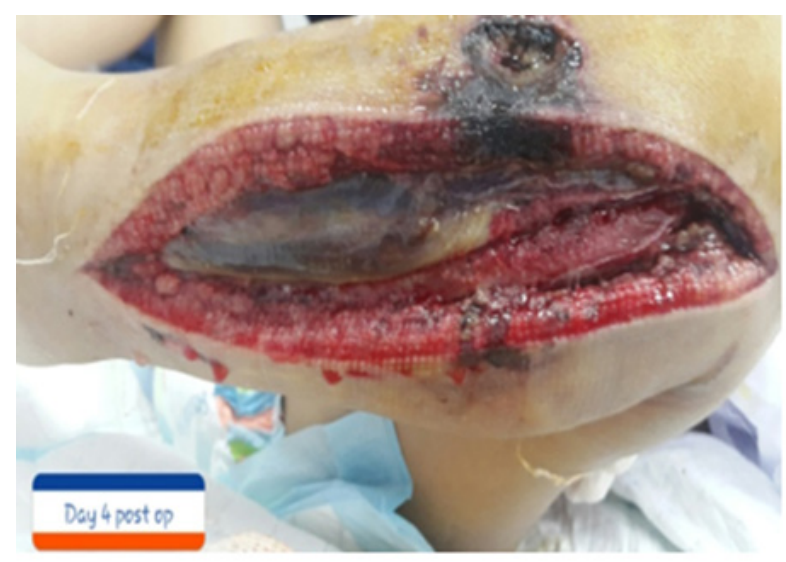

Figure 2 Day 4 post operation.

In Lebanon, around 20 types of snakes are present. Only three types available are poisonous, where V. palastinae, the one mentioned in our case, is one of them. It was reported around 40 cases of snake bites from Akkar, out of which 30\% were reported inside houses ${ }^{7}$ 2016 was revealed to be a particularly hot year with a minimum level of rain. This caused snakes to be more aggressive and to run into houses looking for cold and humidity (Figure 3).

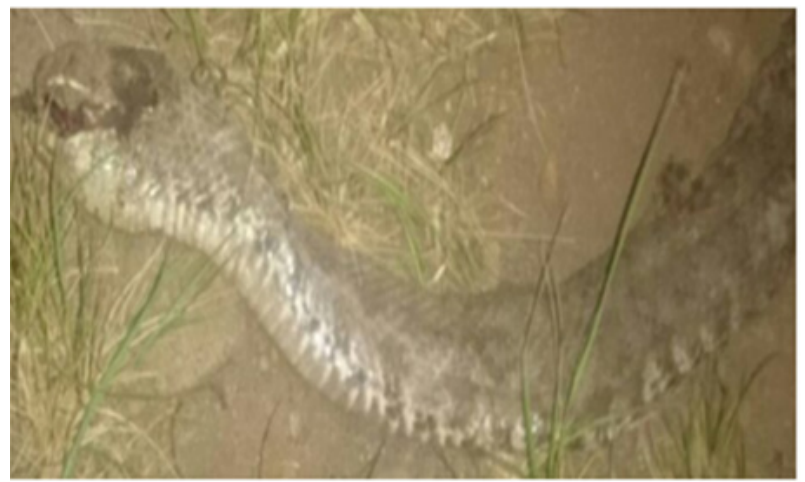

Figure 3 The Snake.
The current management relies on avoiding the following measures that usually causes worsening the patient's condition: using a tight tourniquet, wound incision or ice. In addition there is no benefit of doing an incision and suction of the venom. ${ }^{8}$ In our case, all these steps were done which worsened the situation and led to damage to the leg and probable amputation if she had not been transferred on time to our P.I.C.U. Surgical management of fasciotomy is performed only in cases with compartment syndromes, ${ }^{5}$ as was noted in our patient. In addition, the antivenom should be given within four hours of the bite, with a maximum delay of 24 hours [3]. Besides all the wrong steps that were performed to our patient, the leg was preserved and almost healed. N.B. Of note is that a relative of the daughter had same situation 15 days after this patient.

\section{Conclusion}

Although snakebites and inadequate management can cause fatal consequences, giving the antivenom at the right time and doing fasciotomy can be lifesaving.

\section{Acknowledgements}

None.

\section{Conflicts of interest}

The authors declare no conflict of interest.

\section{Funding}

None.

\section{References}

1. Chippaux JP. Snake bites: Appraisal of the global situation. Bull World Health Organ. 1998;76(5):515-524.

2. Simpson ID. The pediatric management of snakebite the national protocol. Indian Pediat. 2007;44(3):173-176.

3. Ahmed SM, Ahmed M, Nadeem A, et al. Emergency treatment of a snake bite:Pearls from literature. Journal of emergencies, trauma, and shock. 2008;1(2):97.

4. Cavazos MD, Hernández-Montelongo BA, Garza CT, et al. Snake bites in pediatric patients, a current view. INTECH Open Access Publisher. 2012. $10.5772 / 34749$

5. Juckett G, Hancox JG. Venomous snakebites in the United States:management review and update. Am Fam Physician. 2002;65(7):1367-1378.

6. Karunanayake RK, Dissanayake DM, Karunanayake AL. A study of snake bite among children presenting to a paediatric ward in the main Teaching Hospital of North Central Province of Sri Lanka. BMC research notes. 2014;7:482.

7. Haddad N, Salam N, Kazzi Z, et al. Snake bite surveillance in Lebanon, 2010W26-2011W26. Eastern Mediterranean Conference, Egypt. 2011.

8. Stewart ME, Greenland S, Hoffman JR. First-aid treatment of poisonous snakebite:Are currently recommended procedures justified? Ann Emerg Med. 1981;10(6):331-335. 